\title{
DEFICIENTE COBERTURA DE ASEGURAMIENTO A MÉDICOS DURANTE EL SERVICIO RURAL Y URBANO-MARGINAL EN PERÚ
}

\author{
Christian R. Mejia ${ }^{1,2, a}$, Dante M. Quiñones-Laveriano, $2,3, \mathrm{~b}$, Klaudia G. Espinoza,2,3, \\ Claudia Quezada-Osoria ${ }^{4, a}$
}

\begin{abstract}
RESUMEN
Con el objetivo de determinar la cobertura del aseguramiento en salud que recibieron los médicos recién graduados de universidades de Lima, que realizaron el Servicio Rural y Urbano Marginal en Salud (SERUMS) durante el periodo 2012 - 2013, realizamos un seguimiento mensual de los datos de afiliación al Seguro Social de Salud (Essalud). De un total de 290 médicos el 6,6\% (19) tenía activado su seguro al fin de los tres primeros meses, incrementándose al $72 \%$ (209) el último mes, el $26,2 \%$ no tuvieron afiliación en ninguno de los meses. Se encontraron diferencias en la cobertura de aseguramiento según entidad que regula la sede $(p<0,01)$, el sexo del médico $(p=0,03)$ y la clasificación de pobreza de la sede $(p=0,02)$. Se concluye que existe una deficiente cobertura de aseguramiento hacia los profesionales que realizan atención primaria de la salud en zonas alejadas del país, lo cual es un problema que debe ser vigilado.
\end{abstract}

Palabras clave: Seguridad social; Recursos humanos; Trabajadores rurales; Atención primaria de salud (fuente: DeCS BIREME)

\section{POOR COVERAGE INSURANCE IN MEDICAL GRADUATES FOR THE SERVICE RURAL AND URBAN-MARGINAL IN PERÚ}

\begin{abstract}
The aim of this study was to describe the concentration of lead in umbilical cord blood (UCB) of neonates born from primiparous women who live in northern Lima. A cross sectional study was carried out at Cayetano Heredia National Hospital, from July 2011 to January 2012. 100 neonates born from primiparous women who lived in northern Lima in the previous 5 years or more were included. Umbilical cord lead levels were measured by the atomic adsorption method, and the demographic information and risk factors of the neonates were filled in a patient record designed to this purpose. $30 \%$ of the neonates had concentration of lead in UCB that was considered as increased risk ( $\geq 3,0 \mu \mathrm{g} / \mathrm{dL}), 16 \%$ of the cases had had toxic concentration of lead in UCB ( $\geq 5 \mu \mathrm{g} / \mathrm{dL})$. We conclude that there are a high percentage of neonates contaminated with lead in the northern Lima.
\end{abstract}

Key words: Social security; Human resources; Rural workers; Primary health care (source: MeSH, NLM).

\section{INTRODUCCIÓN}

Los profesionales que laboran en atención primaria de la salud (APS) son parte fundamental en el sistema sanitario, por ser los encargados de llevar salud a las poblaciones más vulnerables y alejadas ${ }^{(1-3)}$. En nuestro medio, la mayor parte de los profesionales que realizan APS son los recién egresados que están cumpliendo su
Servicio Rural y Urbano Marginal en Salud (SERUMS) ${ }^{(4,5)}$, siendo la entidad encargada del proceso de adjudicación de sedes es el Ministerio de Salud (MINSA) ${ }^{(6)}$. Para que puedan cumplir adecuadamente su función ${ }^{(7,8)}$, además de brindarles beneficios que mejoren sus capacidades profesionales, cada institución de salud que regula las sedes donde se desempeñan estos profesionales deberá brindar la seguridad social mientras dure su contrato ${ }^{(9)}$.

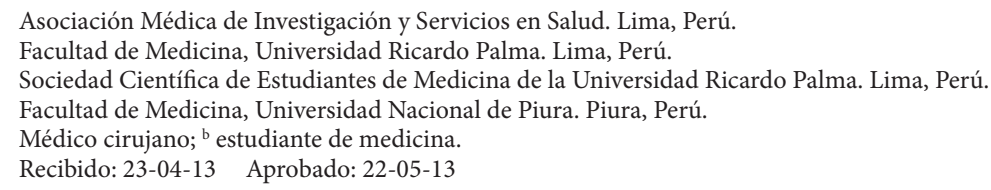

Citar como: Mejía CR, Quiñones-Laveriano DM, Espinoza KG, Quezada-Osoria C. Deficiente cobertura de aseguramiento en médicos durante el servicio rural y urbano-marginal en salud. Rev Peru Med Exp Salud Publica. 2013;30(2):220-3. 
La cobertura de un seguro de salud es esencial en países como el nuestro, donde profesionales que laboran en zonas pobres y extremadamente pobres de las regiones más alejadas del país se encuentran en riesgo de enfermar o sufrir accidentes en el cumplimiento de su labor, evidenciándose, además, una alarmante mortalidad en los recién egresados que realizan el SERUMS $(10,11)$. El objetivo del estudio fue determinar la cobertura del aseguramiento en salud que recibieron los médicos recién graduados de universidades de Lima durante el SERUMS.

\section{EL ESTUDIO}

Se realizó un estudio usando los datos del asesoramiento y seguimiento a los médicos que egresaron de alguna universidad en Lima y que se colegiaron entre enero y abril de 2012 en el Consejo Regional III del Colegio Médico del Perú. El tamaño muestral fue escogido por conveniencia, de un mínimo de 233 participantes, de una población finita de 1800 médicos que se colegian anualmente ${ }^{(12)}$, usando un nivel de significancia del $5 \%$ (alfa 1,96 ), con una precisión del $6 \%$ (beta 0,06 ) y con un estimado de $50 \%$ como promedio de apoyo adecuado recibido. (Epitable, programa en EPINFO, Versión 6.04).

Para obtener los datos del aseguramiento o afiliación al Seguro Social de Salud (SSS) - Essalud usamos los números de documentos de identidad de los recién egresados. Teniendo la premisa que los afiliados -los contratados por el estado o alguna entidad particular- y sus derecho habientes cuentan con el beneficio de las prestaciones del SSS siempre que aquellos cuenten con tres meses de aportación consecutivos o cuatro no consecutivos ${ }^{(13)}$.

Considerando como día de inicio del contrato el primer día de trabajo SERUMS, el 5 de mayo de 2012; se consultó cada mes desde el día 100 (13 de agosto de 2012), a la plataforma virtual de Essalud (http://ww4.essalud.gob. pe:7777/acredita/) para corroborar el estado de aseguramiento, las opciones que obtuvimos fueron: asegurado, no asegurado, y sin información, esta última la tomamos como no asegurado para motivos del análisis, ya que si no figuraba en el sistema significaba que nadie había depositado o hecho el trámite de aseguramiento para esa persona.

Para el análisis descriptivo de las variables cuantitativas usamos la medida de tendencia central y dispersión más recomendada según el análisis previo con la prueba Shapiro Wilk. Las variables categóricas las describimos con frecuencias y porcentajes. Se usó la $U$ de Mann-Whitney para determinar las diferencias entre la cantidad de meses afiliados según las variables categóricas (sexo, entidad contratante -MINSA u otro- y sede catalogada como de extrema pobreza).
Se cuenta con la aprobación del comité de ética del Hospital Nacional San Bartolomé (expediente: CEI1306381). Asimismo, se les informó a los encuestados que sus datos se usarían para el seguimiento y envío de información durante el SERUMS; se contó con el consentimiento de los encuestados.

\section{HALLAZGOS}

De los 290 médicos, 173 (59,7\%) fueron mujeres, la mediana de edad fue 26 años (rango intercuartílico: 2337 años). Aquellos departamentos que tuvieron mayor cantidad de médicos realizando el SERUMS fueron Lima (18,3\%), Huánuco $(10,3 \%)$ y Cajamarca $(10 \%)$.

EI MINSA fue la institución que regulaba el $85 \%$ (239) de las sedes y la misma cantidad adjudicó una plaza remunerada en alguno de los tres procesos de elección del 2012-I. Las otras características se muestran en la Tabla 1.

A los tres meses de iniciado su contrato, el 6,6\% (19) de los médicos recién egresados contaban con Seguro Social en caso de accidentes y muerte, esto fue aumentando conforme pasaban los meses, septiembre $(17,9 \%)$; octubre $(61,7 \%)$; noviembre $(66,2 \%)$; diciembre $(69,7 \%)$; enero $(69,7 \%)$; febrero $(69,7 \%)$; marzo $(71,0 \%)$, hasta llegar a su máximo el último mes de su contrato, abril (72,1\%). Quince (5,2\%) médicos que realizaron atención primaria de la salud tuvieron cobertura de Seguro Social a los nueve meses y $76(26,2 \%)$ no lo tuvieron en ninguno.

Tabla 1. Características de la sede de atención primaria de la salud donde se realizó el Servicio Rural y Urbano Marginal

\begin{tabular}{lr}
\hline Características & \multicolumn{1}{c}{$\mathbf{N .}^{\circ}(\%)$} \\
\hline Entidad que regula la sede & \\
Ministerio de Salud & $239(85,1)$ \\
Policía Nacional del Perú & $11(3,9)$ \\
Ejército Peruano & $9(3,2)$ \\
Marina de Guerra & $8(2,8)$ \\
Fuerza Aérea & $7(2,5)$ \\
Essalud & $6(2,1)$ \\
\hline Particular & $1(0,4)$ \\
Dependencia presupuestaria & $164(61,6)$ \\
Nacional & $60(22,6)$ \\
Regional & $42(15,8)$ \\
No sabe & $131(46,9)$ \\
Evaluación de pobreza de la sede & $102(36,6)$ \\
Extrema pobre & $43(15,4)$ \\
Muy pobre & $1(0,4)$ \\
Pobre & $2(0,7)$ \\
Regular & \\
Aceptable & $149(53,2)$ \\
Fase del proceso en la que eligió sede & $82(29,3)$ \\
1.. elección* & $8(2,9)$ \\
2. ${ }^{a}$ elección* & $41(14,6)$ \\
\hline 3. elección* & \\
Equivalente/no remunerada &
\end{tabular}




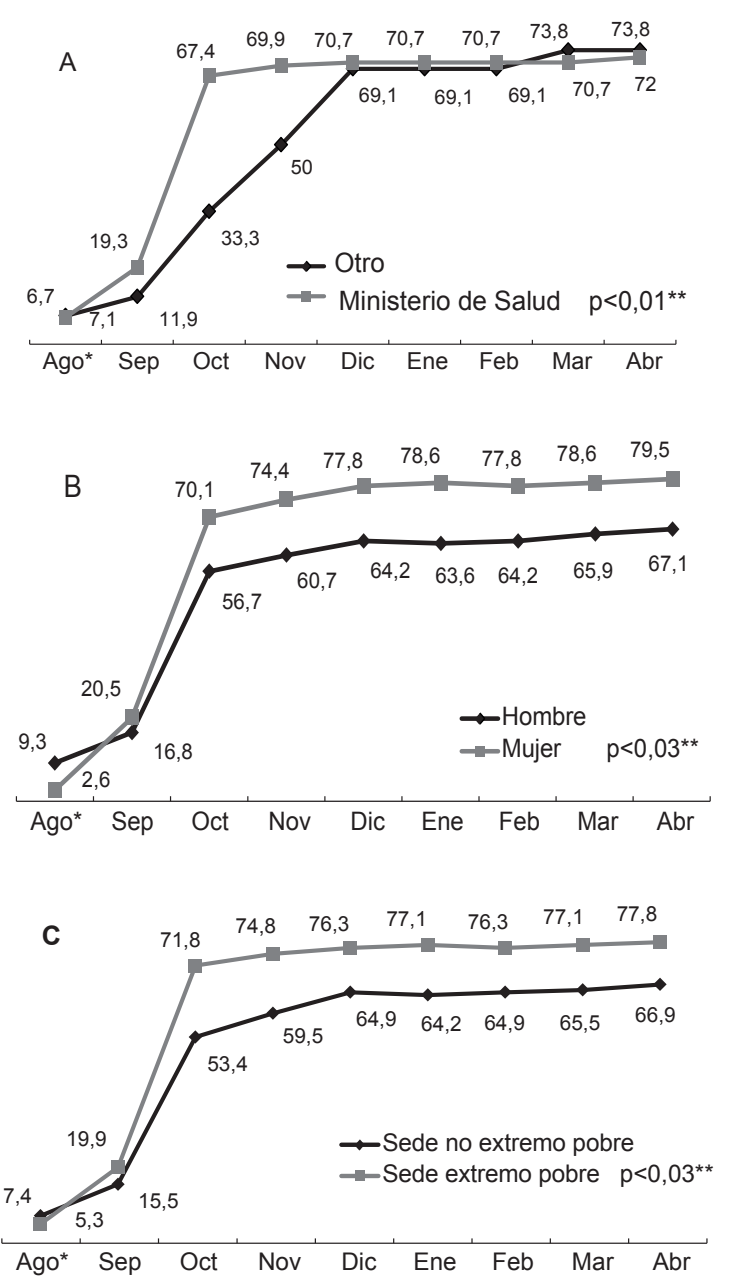

Figura 1. Porcentaje de médicos que realizan el Servicio Rural y Urbano Marginal en Salud con afiliación al Seguro Social de Salud según A. Entidad que regula la sede. B. Sexo. C. Clasificación de pobreza de la sede

* Inicio del tecer mes de contrato, **Prueba U de Mann-Withney.

Se encontró diferencias significativas al realizar el análisis de la afiliación al Seguro Social de Salud según trabajar o no para el MINSA $(p<0,01)$, el sexo $(p=0,03)$ $y$ si la sede fue catalogada como extremo pobreza $(p=0,02)$ Figura 1.

\section{DISCUSIÓN}

Los resultados presentados muestran que uno de cada veinte médicos que realizaron el SERUMS contaba con una adecuada cobertura del Seguro Social de Salud; considerando que en nuestro país los profesionales recién egresados conforman uno de los pilares de la APS ${ }^{(4,5)}$ su salud e integridad física debe ser una prioridad de las instituciones prestadoras de salud (14-17).
Algunos estudios refieren que ítems como la educación médica es inadecuada en las zonas rurales $(9,18,19)$, siendo necesario brindarles los recursos para poder desarrollarse profesionalmente ${ }^{(3,7)}$; sin embargo, no hemos encontrado referencia que precedan a nuestro hallazgo. Según nuestros resultados las coberturas de aseguramiento entre las entidades que regulan la sede, sean del MINSA u otras, el sexo del profesional y la clasificación de pobreza de la sede son significativos con más del $10 \%$ de diferencia a partir del mes de octubre.

Considerado que los profesionales de la salud que laboran en zonas rurales y las más alejadas del país están continuamente expuestos a sufrir enfermedades ocupacionales, así como accidentes $(10,20)$. Uno de los entes rectores, el MINSA, creó hace un tiempo el seguro por incapacidad y muerte para los profesionales SERUMS, esta medida fue un importante paso para la generación de soluciones más eficaces ${ }^{(4,11)}$.

Esta baja y tardía cobertura en el aseguramiento de los médicos que realizan SERUMS podría deberse a una falta de comunicación entre la entidad que regula la sede y la oficina de aseguramiento, o con la dependencia de partida presupuestaria, si bien nuestros resultados solo muestran este problema en médicos egresados de Lima, desconocemos cuál es la situación de los médicos egresados de provincias o aquellos egresados en el extranjero y que realizan SERUMS en Perú, lo cual requiere ser vigilado.

Una limitación del estudio fue no realizar un muestreo aleatorio, esto debido a que las listas oficiales de los profesionales que adjudican alguna plaza SERUMS no son de dominio público, a pesar de esto, se cree que las respuestas de los encuestados pueden ser un buen aproximado de la realidad, ya que se trabajó con un buen nivel de precisión al momento de calcular el tamaño muestral y porque los datos de la muestra son muy similares a los porcentajes de los profesionales egresados de universidades de Lima que realizaron el SERUMS en el proceso anterior.

Se concluye que existe una deficiente cobertura del aseguramiento de aquellos médicos recién egresados de universidades de Lima que realizan el SERUMS. Se espera que este estudio sea un aporte para que las entidades responsables apliquen medidas a corto y mediano plazo que mejoren el apoyo que se les brinda a los médicos que realizarán el SERUMS.

Agradecimientos: los autores agradecen a los integrantes del Grupo de Investigación AMISS-SOCEMURP por sus contribuciones, orientación y sugerencias sobre el diseño del estudio, análisis de datos y preparación de manuscritos de la 
presente publicación. Este artículo fue preparado como uno de los productos del programa antes mencionado.

Contribuciones de autoría: CRM y CQO tuvieron la idea de investigación y diseñaron el estudio, DQL, CKE y CQO recopilaron los datos, CRM realizó el análisis e interpretación de datos, todos los autores participaron en la redacción del manuscrito y aprobaron la versión final a publicar.

Financiamiento: autofinanciado.

Conflicto de interés: los autores declaran no tener conflictos de interés.

\section{REFERENCIAS BIBLIOGRÁFICAS}

1. Martiniuk ALC, Adunuri N, Negin J, Tracey P, Fontecha C, Caldwell P. Primary care provision by volunteer medical brigades in Honduras: a health record review of more than 2,500 patients over three years. Int $\mathrm{J}$ Health Serv. 2012;42(4):739-53.

2. Wouters E, Van Damme W, van Rensburg D, Masquillier C, Meulemans H. Impact of community-based support services on antiretroviral treatment programme delivery and outcomes in resource-limited countries: a synthetic review. BMC Health Serv Res. 2012;12:194.

3. Curry LA, Alpern R, Webster TR, Byam P, Zerihun A, Tarakeshwar N, et al. Community perspectives on roles and responsibilities for strengthening primary health care in rural Ethiopia. Glob Public Health. 2012;7(9):961-73.

4. Arroyo J, Hartz J, Lau M. Recursos Humanos en Salud al 2011: Evidencias para la toma de decisiones. Lima: Ministerio de Salud; 2011.

5. Arroyo J. Los sistemas descentrados de recursos humanos en salud: el caso del Perú, 1990-2005. Cien Saude Colet. 2006;11(4):1063-72.

6. Galán-Rodas E, Rodríguez JA, DíazVélez C et al. Proceso de admisión al Residentado Médico en el Perú: Características, preferencias y propuestas de los médicos que se preparan para rendir el examen de admisión 2011. Acta méd. peruana. 2010;27(4):257-63.

7. Phalkey R, Dash SR, Mukhopadhyay A, Runge-Ranzinger S, Marx M. Prepared to react? Assessing the functional capacity of the primary health care system in rural Orissa, India to respond to the devastating flood of September 2008. Glob Health Action. 2012;5. doi: 10.3402/gha.v5i0.10964. Epub 2012 Mar 14.

8. De Mendonça MHM, Martins MIC, Giovanella L, Escorel S. Desafios para gestão do trabalho a partir de experiências exitosas de expansão da Estratégia de Saúde da Família. Cien Saude Colet. 2010;15(5):2355-65.

9. Hana J, Rudebeck CE. Leadership in rural medicine: the organization on thin ice? Scand J Prim Health Care. 2011;29(2):122-8.

10. Galán-Rodas E, Díaz-Vélez C, Villena J, Maguiña C. Mortalidad de médicos que Realizan el servicio rural (SERUMS) en Perú, 2006-2009. Rev Peru Med Exp Salud Publica. 2010;27(3):483-4.

11. Curisinche $M$, Yagui $M$, Castilla $T$. Proceso de construcción de la agenda nacional de investigación sobre recursos humanos en salud (RHUS) en el Perú, 2011-2014. Rev Peru Med Exp Salud Publica. 2011;28(2):372-81.

12. Mayta-Tristán P, Mejia C, RiegaLopez P, Rojas Mezarina L, Posso M, Mezones-Holgín E. Proyección de trabajo en el interior del país y factores asociados en médicos recién colegiados de Lima, Perú 2010. Rev Peru Med Exp Salud Publica. 2011;28(2):186-93.

13. Congreso de la República del Perú. Ley de Modernización de la Seguridad Social en Salud. Ley No 26790. 15 de mayo de 1997.

14. Beatty K, Harris JK, Barnes PA. The role of interorganizational partnerships in health services provision among rural, suburban, and urban local health departments. J Rural Health. 2010;26(3):248-58.

15. Kalita A, Zaidi S, Prasad V, Raman VR. Empowering health personnel for decentralized health planning in India: The Public Health Resource Network. Hum Resour Health. 2009; 7:57.

16. Montoya-Aguilar C. Atención primaria de salud. Alma Ata otra vez y la experiencia de Chile. Médicos Sociales. 2008;48(3):147-54.

17. Tuan T, Dung VTM, Neu I, Dibley MJ. Comparative quality of private and public health services in rural Vietnam. Health Policy Plan. septiembre de 2005;20(5):319-27.

18. Jerome G, Ivers LC. Community health workers in health systems strengthening: a qualitative evaluation from rural Haiti. AIDS. 2010;24 Suppl 1:S67-72.

19. Manafa O, McAuliffe E, Maseko F, Bowie C, MacLachlan M, Normand C. Retention of health workers in Malawi: perspectives of health workers and district management. Hum Resour Health. 2009;7:65.

20. Galán-Rodas E, Gálvez-Buccollini JA, Vega-Galdós F. Salud mental en médicos que realizan el servicio rural, urbano-marginal en salud en el Perú: Un estudio de línea base. Rev Peru Med Exp Salud Publica. 2011;28(2):277-81.

\section{Correspondencia: Christian Mejia.}

Dirección: Av. Las Palmeras 5713 - Los

Olivos. Lima 39 - Perú.

Teléfono: (511) 997643516.

Correo electrónico: christian.mejia.md@ gmail.com 